\title{
Postorthodontic Relapse Prevention by Administration of Grape Seed (Vitis vinifera) Extract Containing Cyanidine in Rats
}

\author{
Ananto Ali Alhasyimi ${ }^{1} \quad$ Niswati Fathmah Rosyida ${ }^{1} \quad$ Mufliha Santi Rihadini ${ }^{1}$ \\ ${ }^{1}$ Department of Orthodontics, Faculty of Dentistry, Universitas \\ Gadjah Mada, Yogyakart, Indonesia \\ Address for correspondence Ananto Ali Alhasyimi, DDS, MDSc, PhD, \\ Department of Orthodontics, Faculty of Dentistry, Universitas Gadjah \\ Mada, Yogyakarta 55281, Indonesia (e-mail: anantoali@ugm.ac.id).
}

Eur J Dent 2019;13:629-634

\begin{abstract}
Keywords

Objective The aim of this study was to analyze the effect of grape seed extract containing cyanidin on osteoclastogenesis (by means of receptor activator of nuclear factor- $\kappa$ B ligand [RANKL] and osteoprotegerin [OPG] levels) and the number of osteoclasts during orthodontic relapse in Wistar rats.

Materials and Methods This study is an in vivo quasi experimental research. A total of 32 male Wistar rats were used in the study, which were randomly split equally into two groups, grape seed (GS) and control group (CG). All rats were given an orthodontic force of $35 \mathrm{cN}$ using a stainless steel 3-spin coil spring that was activated for 7 days and then conditioned to be passive. During this phase, the GS group was administered grape seed extract containing cyanidin once per day. Orthodontic appliances were removed from both groups afterward, and then the alveolar bone tissue was isolated consecutively according to observation days (days 1, 3, 7, and 14), while OPG and RANKL levels were analyzed in their gingival crevicular fluid using a specific enzymelinked immunosorbent assay (ELISA). Tissues were then stained with hematoxylineosin (H\&E) and observed under a light microscope to count the number of osteoclast cells. Data were analyzed statistically using an independent $t$-test $(p<0.05)$.

Results The number of osteoclasts in the GS group was significantly lower than that in the CG group on all experiment days $(p=0.021 ; p=0.001 ; p=0.024 ; p=0.001 ; p<0.05)$. ELISA results showed that the RANKL level of the GS group was significantly lower on

- orthodontic relapse

- grape seed extract

- cyanidin

- osteoclast

- osteoprotegerin days 3 and $7(p=0.025 ; p=0.039 ; p<0.05)$, while the OPG level was significantly higher on days 1 and 3 in the GS group than in the CG group ( $p=0.039 ; p=0.021 ; p<0.05$ ).

Conclusion Grape seed extract can prevent postorthodontic relapse movement by inhibiting osteoclastogenesis and reducing the number of osteoclasts in Wistar rats.
\end{abstract}

\section{Introduction}

Orthodontic treatment is a procedure that straightens the teeth to obtain good aesthetic and normal occlusion function. ${ }^{1}$ In orthodontic treatment, the most common problem faced by an orthodontist is orthodontic relapse, which could be caused by a long and unstable process of bone remodeling. ${ }^{2}$ Bone remodeling is a complex continuous process of bone destruction and renewal and consists of three consecutive phases, one among which one is bone resorption by osteoclasts. The osteoclast is a cell whose function is to resorb trabecular bone during bone growth. ${ }^{3}$ Osteoclast formation, known as osteoclastogenesis, is induced by the receptor acti-

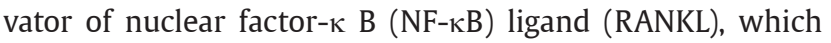
induces the transcription factors of osteoclasts. ${ }^{4}$ During orthodontic relapse, there is an increase in RANKL levels but a decrease in osteoprotegerin (OPG) levels. ${ }^{5}$ 
Prevention of orthodontic relapse is attempted mechanically using a retainer and pharmacologically using drugs such as bisphosphonates. However, either the retainer or bisphosphonates induce other effects in the patients. Relapse could still occur 10 years after retainer discharge, and bisphosphonate use is associated with side effects such as oral intolerance and jaw bone osteonecrosis. ${ }^{6,7}$ Therefore, natural remedies might be an alternative to reduce those effects. ${ }^{8}$

Grape seeds are a natural source of proanthocyanidins, one of the phenolic compounds. Furthermore, one of the proanthocyanidin members, procyanidin, forms a complex known as cyanidin. Cyanidin has three forms of groups, one of which is primarily found in a glycosylated form known as cyanidin-3-glucoside (C3G). ${ }^{9}{ }^{10}$ Studies have found that C3G has the potential to inhibit osteoclast differentiation by reducing the activation of mitogen-activated protein kinases (MAPKs) induced by RANKL at a dose higher than $10 \mu \mathrm{g} / \mathrm{ml}$. In addition, it was found to significantly reduce the expression of the c-Fos and nuclear factor of activated T-cells (NFATc1) genes, the primary transcription factors of osteoclastogenesis. ${ }^{11-13}$ There is no study that analyzes the potential of grape seed extract containing cyanidin toward osteoclastogenesis inhibition, which could, in turn, affect the number of osteoclast during orthodontic relapse in rats. The hypothesis of this study was that grape seed extract containing cyanidin would prevent postorthodontic relapse by inhibiting osteoclastogenesis and reducing the number of osteoclasts in Wistar rats.

\section{Materials and Methods}

\section{Determination of Cyanidin in Grape Seeds using Fourier-transform Infrared Spectroscopy}

Validation examinations of functional groups conducted by Fourier-transform infrared (FTIR) spectroscopy using grape seed extract samples demonstrated the successful identification of cyanidin in the samples. The FTIR spectra revealed the presence of a band extending centrally at $3422.63 \mathrm{~cm}^{-1}$, which was a hydroxyl group. At the wavelength $2926.05 \mathrm{~cm}$, the highest transmission peak was detected. The absorption band observed at $1745.19 \mathrm{~cm}^{-1}$ was an extension of the aldehyde group ( - Fig. 1).

\section{Animal and Experimental Procedures}

Ethical clearance was obtained from the Medical and Health Research Ethics Committee, Faculty of Medicine, Universitas Gadjah Mada (UGM), with the approval number KE/FK/0359/ EC/2019. The study animals were 32 healthy male adult Wistar rats (Rattus norvegicus) aged 2.5 to 3 months with body weight of 200 to $250 \mathrm{~g}$. The animals were maintained in polycarbonate cages and fed a standard pellet and tap water ad libitum. Standard environmental conditions of 12-hour, light-dark cycles, temperature of $21^{\circ} \mathrm{C} \pm 2^{\circ} \mathrm{C}$, and humidity level of $50 \% \pm 15 \%$ were maintained. Animals were categorized equally into control and treatment groups (each group containing 16 animals) and were then randomly divided into four subgroups, each with four animals, corresponding to four observation periods, that is, days $1,3,7$, and 14 on the retention stage after the debonding of the appliances.

The orthodontic appliances were applied to all the animals. During this application process, all the animals were anesthetized by intramuscular injections of $0.05 \mathrm{ml} / 100 \mathrm{~g}$ of ketamine (Kepro, Kepro B.V., the Netherlands) and $0.05 \mathrm{ml} / 100 \mathrm{~g}$ of xylazine (Xyla; Interchemie, the Netherlands) mixed in a 1:1 ratio. The appliances were made of $0.012-\mathrm{cm}$ stainless steel U-shaped wire (3M orthodontics, United States), $5 \mathrm{~mm}$ in length with a 2-mm diameter coil; these devices were applied on the maxillary incisors of the animals ( - Fig. 2). The appliances were activated (by bent opening the loop

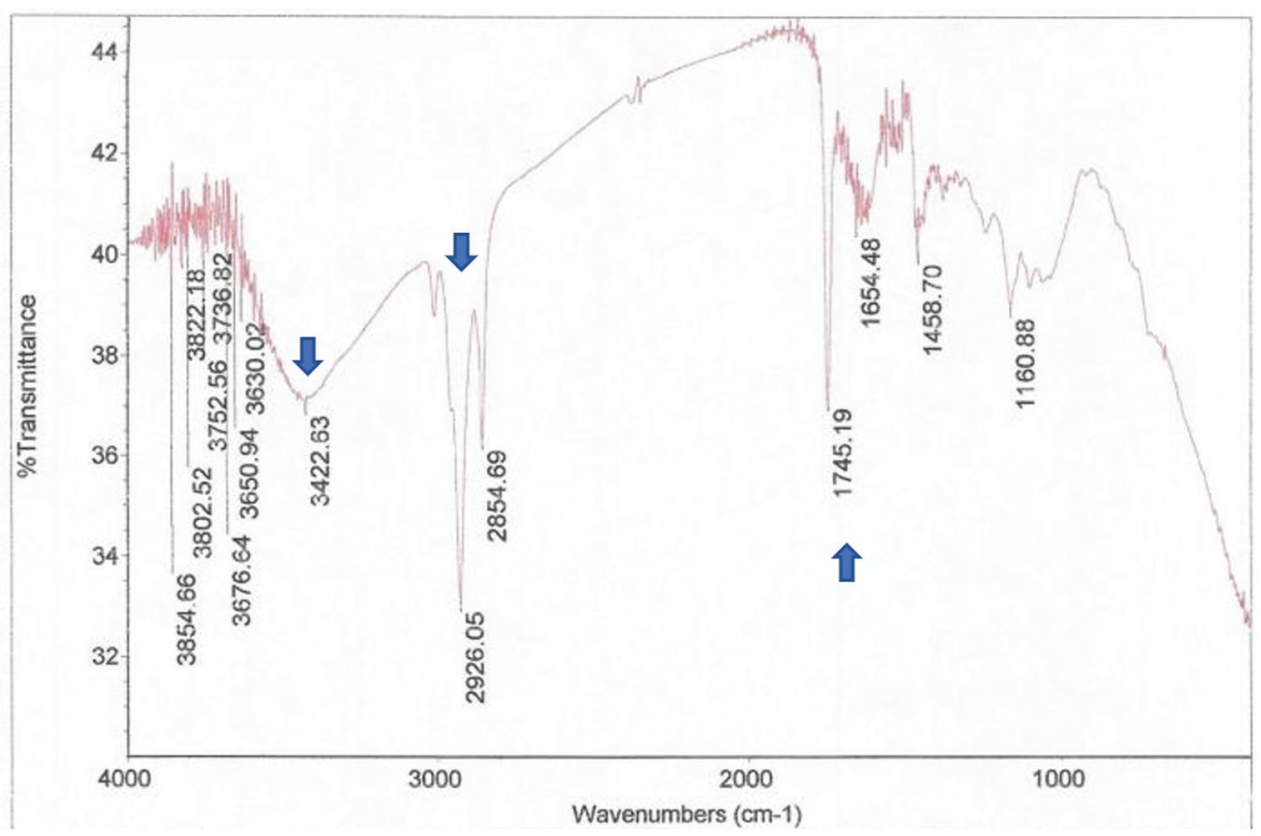

Fig. 1 FTIR spectroscopy analysis results of grape seed extract. The peak wave number was confirmed as a cyanidin spectrum (arrowhead). FTIR, Fourier-transform infrared. 

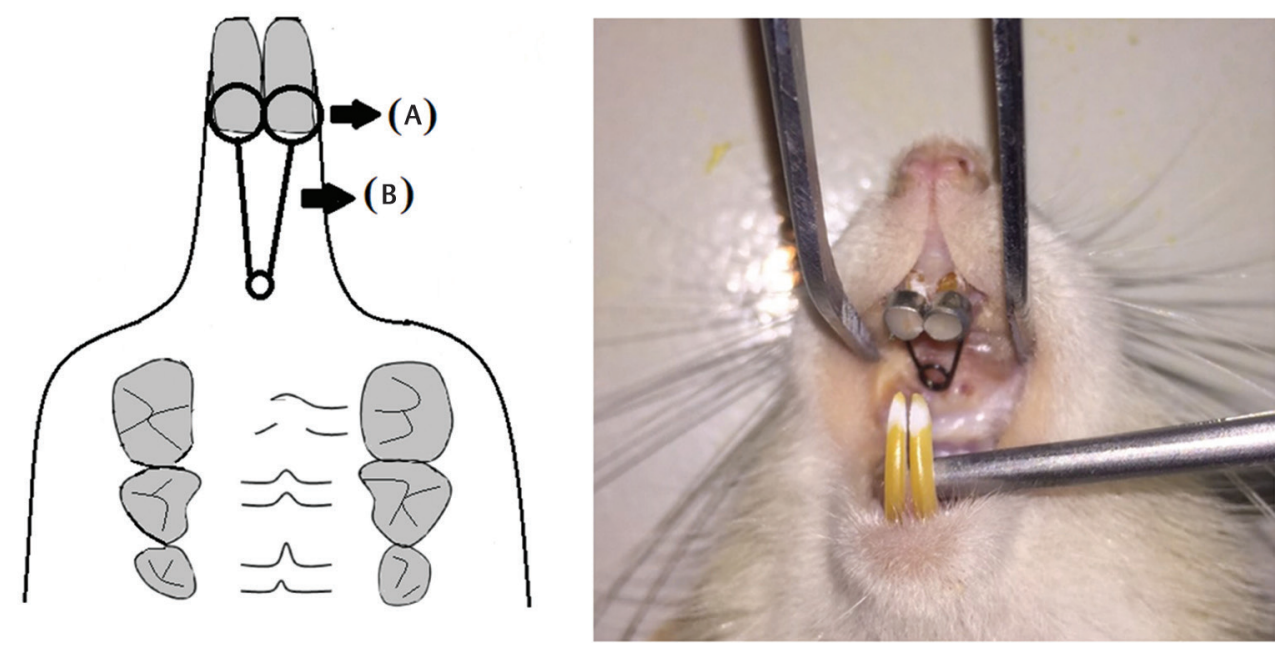

Fig. 2 Design of experimental tooth movement model in rats: (A) matrix band and (B) stainless steel wire.

until it given a standard force of $30 \mathrm{~g}$ force measured using a tension gauge, Medkraft Orthodontics, United States) for 7 days and were then not reactivated to mimic the retention stage for 7 days; the teeth were not moved anymore (known by comparing the incisive distance). In the passive stage, the grape seed extract containing cyanidin was administered orally using oral sonde (Thermo Fisher Scientific, United States) to the animals in the treatment group at a dose of 25 $\mathrm{mg}$ dissolved in $2 \mathrm{ml}$ saline once a day. The appliances were then debonded to allow relapse movement.

\section{Gingival Crevicular Fluid Collection and Enzyme-linked Immunosorbent Assay}

Gingival crevicular fluid (GCF) was obtained using a sterile paper point number 20 (Paper Points ISO 0.2; Dentsply, Germany) on days 1, 3, 7, and 14 after debonding. To prevent contamination of the fluid by the remaining saliva and other oral fluids during GCF isolation, the sulcus of each animal was evaporated mildly by soft air jets and covered with sterile cotton pellets. A paper point was entered into the gingival sulcus (on the pressure side of relapse movement, the mesial sulcus) for 60 seconds. Subsequently, the paper point was placed into a 1.5-mL Eppendorf tube containing $350 \mu \mathrm{L}$ of physiological saline solution and centrifuged for 3 minutes at $2500 \mathrm{rpm}$ to elute the GCF component completely. The GCF was then analyzed for RANKL and OPG levels using the ELISA kit reagent test (Cusabio, China). The levels of RANKL and OPG in the sample were defined by comparing the optical density of the sample with the standard curve using a spectrophotometry microplate reader at $450 \mathrm{~nm}$ wavelength. The final results of RANKL and OPG levels were reported as $\mathrm{pg} / \mathrm{mL}$.

\section{Histological Preparation}

Tissues were retrieved by cervical dislocation in sedated rats (using ketamine and xylazine injections) in the control and treatment groups on days $1,3,7$, and 14 . The rats were then stretched on a surgical board for dissection using surgical scissors by cutting the region of the maxillary incisors with Knable pliers. The tissue pieces were rinsed with $0.9 \% \mathrm{NaCl}$ and stored in a tubular bottle containing $10 \%$ formalin for
24 hours to protect the cell structure. The remaining fixation material was rinsed with running water for 1.5 hours.

Decalcification was performed in a decalcification solution using $10 \%$ ethylenediamine tetraacetic acid (EDTA) (Sigma-Aldrich, United States), repeated twice for a month until the specimen became soft and could be cut. Then, it was rinsed with running water for 1.5 hours to remove the remaining solution. Tissue dehydration was done using alcohol for 60 minutes. The clearing process was conducted use xylol twice for 60 minutes to clean the tissues. Impregnation was done in the infiltration stage of embedding material in the tissues using liquid paraffin at $480^{\circ} \mathrm{C}$. The embedding step was carried out using paraffin, and then the blocking step was conducted with blocks containing hard paraffin and left for a day. The purpose of paraffin (section) block cutting was to prepare histological specimens by attaching the paraffin blocks to the holder and cutting them to a thickness of 4 to $6 \mu \mathrm{m}$ in a rotary microtome lengthwise on the next day. The mounting process was performed on a glass slide with $5 \%$ gelatin.

Hematoxylin-eosin (H\&E) staining was performed to observe the number of osteoclasts histologically. Deparaffination was done by entering paraffin blocks into xylol, which were performed two times each in 3 minutes. The rehydration step was conducted with water using a graded alcohol series of $70 \%, 80 \%$, and $90 \%$ each for 3 minutes, and then the samples were rinsed with running water for 1 minute. Hematoxylin staining was performed for 6 to 7 minutes before rinsing the samples with running water for 1 minute. Then, eosin solution staining was done for 3 minutes before rinsing the samples with running water for 1 minute, followed by rinsing with $70 \%$ alcohol, and a final rinsing with water for 1 minute until the samples dried. Then, the mounting process was performed with the mounting solution Entellan and covered with a coverglass. The remaining melted Entellan was cleaned using a cotton swab that was previously moistened with xylol. Finally, the dried preparation was labeled.

\section{Histological Analysis}

Histological analysis was conducted on the pressure side of relapse movement (mesial), and 6 fields from the cervical 
to the apical region of alveolar bone were selected at random as region of interests (ROIs). The number of osteoclasts were obtained by observing the specimens under a light microscope equipped with a digital camera (Optilab LLC Phoenix, United States) at $400 \times$ magnification. The number of osteoclasts per field was calculated using Image Raster 3.0 (United States). Histological evaluation was performed by three blinded investigators and repeated thrice. The examiners showed a good level agreement in their

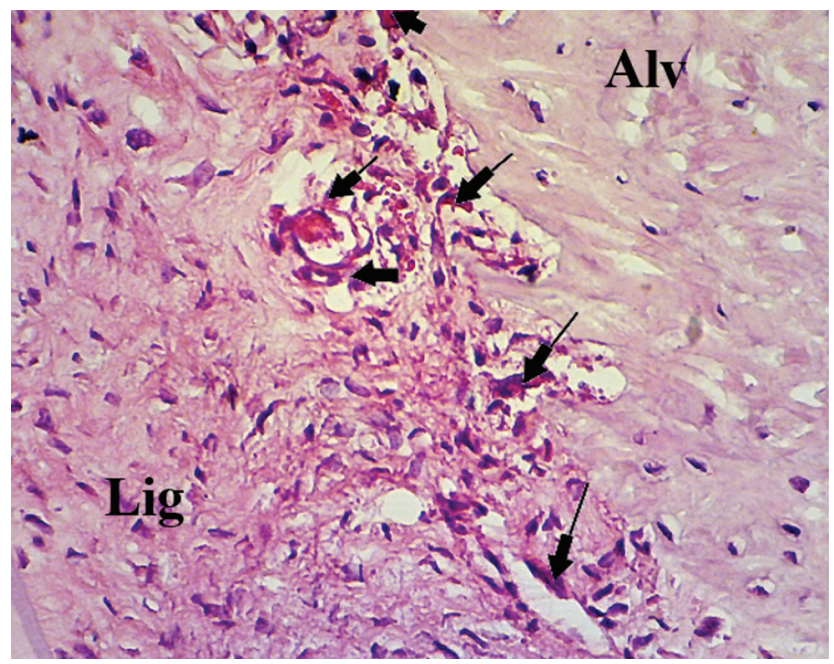

Fig. 3 Osteoclasts stained with H\&E. Alv indicates alveolar bone; Lig indicates periodontal ligament. $\mathrm{H \& E}$, hematoxylin-eosin. analysis $(\kappa=0.89)$, indicating satisfactory intra-examiner and inter-examiner reliability. The total number of osteoclasts was obtained by calculating the mean values across 6 ROIs from both incisors. The osteoclasts stained with HE appeared as large, multinucleated cells with pink-stained cytoplasm and were located near Hoswhip lacunae (-Fig. 3).

\section{Statistical Analysis}

Data analysis was performed using parametric tests. The Shapiro-Wilk normality test and Levene's test of homogeneity were used for data analysis with a significance level set at 95\% ( $p>0.05)$. An independent sample parametric t-test was used to compare data between the control and treatment groups, with a significance level of $p<0.05$.

\section{Results}

- Table 1 shows the mean and standard deviation values of the number of osteoclasts, RANKL level, and OPG level between the control and treatment groups (grape seed extract containing cyanidin). Significant differences were observed in the number of osteoclasts when compared among days 1, 3, 7 , and 14. Histological evaluation showed the difference in the number of osteoclasts among two groups tested ( - Fig. 4). The ELISA results demonstrated that the RANKL level of the grape seed extract group was significantly lower on days 3 and 7 . In contrast, the OPG level was significantly higher on days 1 and 3 in the grape seed group than in the control group during orthodontic relapse $(p<0.05)$.

Table 1 Means and standard deviations of osteoclast count (cells/field), RANKL, and OPG levels (pg/mL) among two groups on day $1,3,7$, and 14

\begin{tabular}{|c|c|c|c|}
\hline $\begin{array}{l}\text { Observation time } \\
\text { (Day) }\end{array}$ & Control group (CG) & Grape seed group (GS) & $p$-Value ${ }^{a}$ \\
\hline \multicolumn{4}{|l|}{$\begin{array}{l}\text { Osteoclast count } \\
\text { (cells/field) }\end{array}$} \\
\hline 1 & $19.121 \pm 2.628$ & $14.008 \pm 2.703$ & $0.021^{*}$ \\
\hline 3 & $24.003 \pm 3.081$ & $17.872 \pm 1.935$ & $0.001^{*}$ \\
\hline 7 & $19.122 \pm 2.755$ & $14.372 \pm 1.627$ & $0.024^{*}$ \\
\hline 14 & $16.625 \pm 2.752$ & $10.379 \pm 1.942$ & $0.001^{*}$ \\
\hline \multicolumn{4}{|l|}{ OPG levels (pg/mL) } \\
\hline 1 & $25.259 \pm 3.067$ & $31.676 \pm 4.068$ & $0.039^{*}$ \\
\hline 3 & $28.162 \pm 4.052$ & $35.378 \pm 6.069$ & $0.021^{*}$ \\
\hline 7 & $26.268 \pm 6.064$ & $24.695 \pm 5.051$ & 0.078 \\
\hline 14 & $31.843 \pm 5.102$ & $31.374 \pm 5.095$ & 0.089 \\
\hline \multicolumn{4}{|l|}{ RANKL levels (pg/mL) } \\
\hline 1 & $23.357 \pm 2.043$ & $22.551 \pm 4.032$ & 0.086 \\
\hline 3 & $27.185 \pm 3.023$ & $23.206 \pm 3.036$ & $0.025^{*}$ \\
\hline 7 & $22.471 \pm 4.016$ & $19.742 \pm 2.013$ & $0.039^{*}$ \\
\hline 14 & $20.124 \pm 3.028$ & $21.215 \pm 3.035$ & 0.128 \\
\hline
\end{tabular}

Note: Values are presented as mean \pm standard deviation.

aTested by independent $t$-test of variance.

${ }^{*} p<0.05$, significant differences between the groups.

Abbreviations: OPG, osteoprotegerin; RANKL, receptor activator of nuclear factor-kB ligand. 


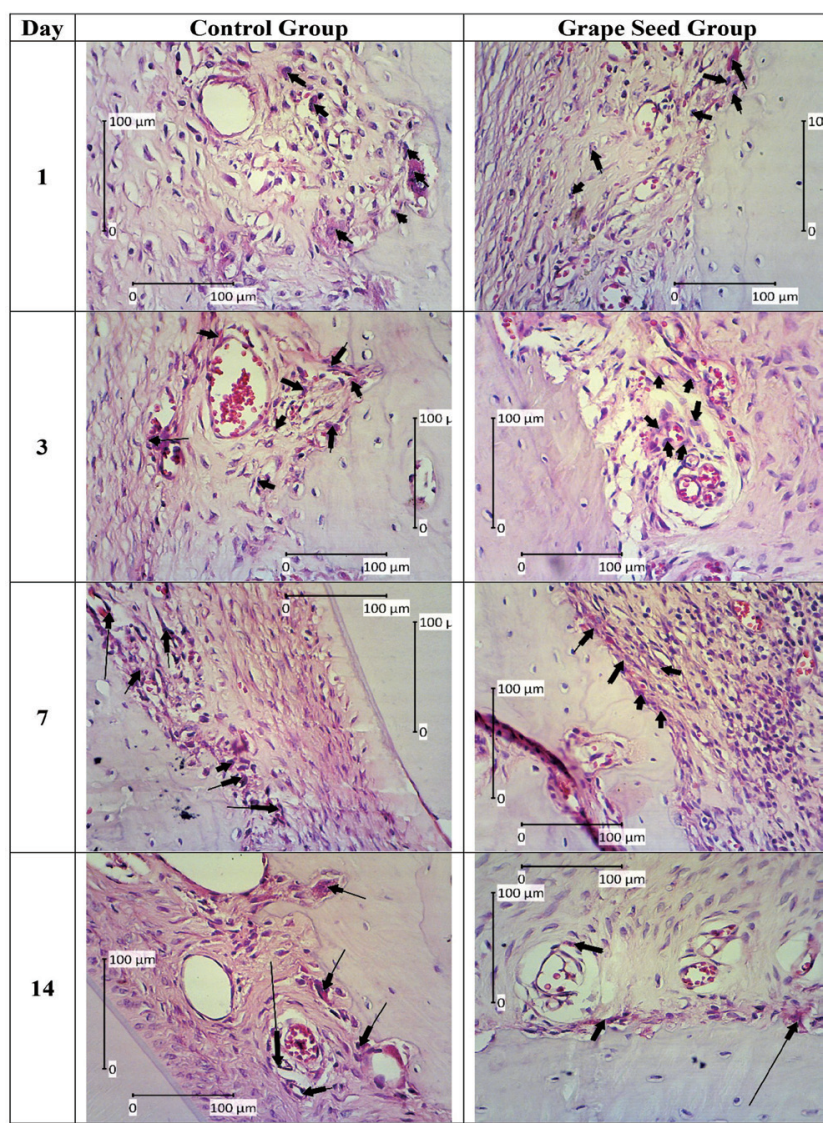

Fig. 4 Osteoclasts stained with H\&E on days 1, 3, 7, and 14 in both the control and treatment groups. The difference could be found in both groups by observing the number of osteoclasts shown in the images (indicated by black arrow; 400× magnification). H\&E, hematoxylin-eosin.

\section{Discussion}

This investigation confirms the hypothesis that grape seed extract containing cyanidin could reduce the number of osteoclasts and block the process of osteoclastogenesis during orthodontic relapse. Results of the independent $t$-test demonstrated a significant difference between the control group and the group treated with grape seed extract containing cyanidin. The grape or Vitis vinifera is known to possess abundant flavonoids, which is a subclass of polyphenolic compounds. Polyphenolic components in grapes could include anthocyanins, flavan-3-ol monomers and proanthocyanidin, phenolic acid (gallic acid), hydroxycinnamate, resveratrol trace, and also flavonols. ${ }^{13}$ It has been reported that the grape seed has several benefits for health as it possesses antidiabetic, antioxidant, antiplatelet, anticholesterol, anti-inflammatory, antiaging, antimicrobial, and antitumoral properties. It contains polyphenolic compounds in the forms of flavan-3-ol, proanthocyanidin (which comprises $90 \%$ of grape seed), and catechins. ${ }^{14,15}$ Proanthocyanidins and catechins are free radical scavengers required in calcium absorption. One of the proanthocyanidin groups, procyanidin, is primarily found in grape seed and can be transformed into cyanidin through an autooxidation process. Cyanidin has some forms, one of which is C3G. It is most abundantly found and known as glycosylated cyanidin.9,10,16 This form of cyanidin can inhibit osteoclast differentiation and formation. It works by inducing c-Fos and NFATc1 as a primary transcription factor of osteoclastogenesis. This process induced by C3G causes significant reduction in the activation of MAPKs (ERK, JNK, and p38); also, induced by RANKL whose effects are significant on p38. ${ }^{11,12}$

The results of this study showed a significant difference in the total number of osteoclasts between the two groups, with the mean osteoclast number being lower in the treatment group than in the control group. This could be caused due to the inhibition of the osteoclastogenesis transcription factor, which inhibited osteoclast formation and differentiation; thus, the total number of osteoclasts was reduced. Previous research has demonstrated that arthritis-induced rats administered $100 \mathrm{mg} / \mathrm{kg}$ dosage of grape seed extract showed a decrease in the expression of the NFATc1 factor, which suppresses osteoclast differentiation. ${ }^{17}$ Cyanidin given at a high dosage $(>10 \mu \mathrm{g} / \mathrm{mL})$ could inhibit RANKL-induced osteoclastogenesis by inhibiting the transcription factors. ${ }^{18}$

Grape seed contains another phenolic compound, the flavonoids. ${ }^{14}$ Flavonoids have anti-inflammatory properties to fight against bone loss. They also have antioxidant properties, which is a defense mechanism for the body to fight against reactive oxygen species produced by normal oxygen metabolism. ${ }^{19}$ These antioxidant properties were found to affect the process of bone remodeling which, in turn, could accelerate bone healing. ${ }^{20}$ Bone remodeling involves the coordination of activities of two cells, that is, osteoblasts in bone synthesis and osteoclasts in bone resorption. ${ }^{1}$ Osteoclasts act in the resorption phase of bone remodeling, which occurs for a period of 2 weeks. Osteoclasts approach the bone surface and adhere to it and then induce the process of resorption, which is completed by osteoclast cell death. This process requires the expression of RANKL to induce osteoclastogenesis transcription factors such as MAPKs, TNF, NF- $\mathrm{kB}, \mathrm{c}-\mathrm{Fos}$, and NFATc1. ${ }^{21}$

Cyanidin is known to inhibit RANKL-induced osteoclastogenesis by decreasing the expression of the transcription factors c-Fos and NFATc 1 and also suppressing MAPK activation. ${ }^{10}$ Upregulation of RANKL is associated with downregulation of OPG, which is expressed from osteoblasts. It acts as a circulating decoy receptor of RANKL. The ratio of RANKL/OPG changes in favor of osteoclastogenesis, which is also a major determinant of bone mass. The relative ratio of RANKL/OPG regulates the differentiation and function of osteoclasts and determines the bone remodeling status. ${ }^{22}$ Based on our study, it was found that the grape seed extract containing cyanidin reduced RANKL expression level and increased the level of OPG. Decreasing the RANKL/OPG ratio may affect osteoclast activity during bone remodeling or vice versa. Increasing the level of OPG could inhibit orthodontic relapse, although it acts as a decoy receptor of RANKL. Increasing the OPG level also enhances osteoblast formation, which helps bone formation in the process of bone remodeling. This process tends to help bone formation in postorthodontic movement, which also prevents the occurrence of orthodontic relapse. 


\section{Conclusions}

Grape seed extract containing cyanidin can prevent postorthodontic relapse by preventing osteoclastogenesis and reducing osteoclast number rats. This result indicates that using the grape seed extract containing cyanidin is a safe and effective pharmacological method to control osteoclast locally for purposes such as the prevention of orthodontic relapse. Further studies are required to establish the effects of different dosages of this treatment on the number of osteoclasts and its efficacy in humans.

\section{Funding}

This work was supported by Universitas Gadjah Mada through Hibah Rekognisi Tugas Akhir in the fiscal year 2019 under the contract No. 3090/UN1/DITLIT/DIT-LIT/LT/2019.

\section{Conflict of Interest}

None declared.

\section{Acknowledgment}

Authors would like to thank the Department of Orthodontic, Faculty of Dentistry, Universitas Gadjah Mada.

\section{References}

1 Narmada IB, Husodo KRD, Ardani IGAW, Rahmawati D, Nugraha AP, Iskandar RPD. Effect of vitamin D during orthodontic tooth movement on receptor activator of nuclear factor kappa- $\beta$. ligand expression and osteoclast number in pregnant wistar rat (Rattus novergicus). JKIMSU 2019;8(1):37-42

2 Alhasyimi AA, Pudyani PP, Asmara W, Ana ID. Enhancement of post-orthodontic tooth stability by carbonated hydroxyapatite-incorporated advanced platelet-rich fibrin in rabbits. Orthod Craniofac Res 2018;21(2):112-118

3 Narmada IB, Rubianto M, Putra ST. The role of low-intensity biostimulation laser therapy in transforming growth factor $\beta 1$, bone alkaline phosphatase and osteocalcin expression during orthodontic tooth movement in cavia porcellus. Eur J Dent 2019;13(1):102-107

4 Al-Rawi NH, Al-Siraj AK, Majeed AH. Comparison of osteoclastogenesis and local invasiveness of ameloblastoma and keratocystic odontogenic tumor. Eur J Dent 2018;12(1):36-42

5 Alhasyimi AA, Pudyani PS, Asmara W, Ana ID. Locally inhibition of orthodontic relapse by injection of carbonated hydroxyapatite advanced platelet-rich fibrin in a rabbit model. Key Eng Mater 2017;758:255-263

6 Al Yami EA, Kuijpers-Jagtman AM, van 't Hof MA. Stability of orthodontic treatment outcome: follow-up until
10 years postretention. Am J Orthod Dentofacial Orthop 1999;115(3):300-304

7 Kennel KA, Drake MT. Adverse effects of bisphosphonates: implications for osteoporosis management. Mayo Clin Proc 2009;84(7):632-637, quiz 638

8 Suparwitri S, Rosyida NF, Alhasyimi AA. Wheat seeds can delay orthodontic tooth movement by blocking osteoclastogenesis in rats. Clin Cosmet Investig Dent 2019;11:243-249

9 Xia E, He X, Li H, Wu S, Li S, Deng G. Biological activities of polyphenols from grapes. Polyphenols Hum Heal Dis. 2013;1:47-58

10 Kong JM, Chia LS, Goh NK, Chia TF, Brouillard R. Analysis and biological activities of anthocyanins. Phytochemistry 2003;64(5):923-933

11 Fang J. Bioavailability of anthocyanins. Drug Metab Rev 2014;46(4):508-520

12 Park KH, Gu DR, So HS, Kim KJ, Lee SH. Dual role of cyanidin-3-glucoside on the differentiation of bone cells. J Dent Res 2015;94(12):1676-1683

13 Li X, Udagawa N, Itoh K, Suda K, Murase Y, Nishihara T, et al. p38 MAPK-mediated signals are required for inducing osteoclast differentiation but not for osteoclast function. Endocrinology 2002;143(8):3105-3113

14 Vislocky LM, Fernandez ML. Biomedical effects of grape products. Nutr Rev 2010;68(11):656-670

15 Ma Z, Zhang H. Phytochemical constituents, health benefits, and industrial applications of grape seeds: a mini-review. Antioxidants 2017;6(3):71-77

16 Yahara N, Tofani I, Maki K, Kojima K, Kojima Y, Kimura M. Mechanical assessment of effects of grape seed proanthocyanidins extract on tibial bone diaphysis in rats. J Musculoskelet Neuronal Interact 2005;5(2):162-169

17 Park JS, Park MK, Oh HJ, Woo YJ, Lim MA, Lee JH, et al. Grape-seed proanthocyanidin extract as suppressors of bone destruction in inflammatory autoimmune arthritis. PLoS One 2012;7(12):e51377

18 Dou C, Li J, Kang F, Cao Z, Yang X, Jiang H, et al. Dual effect of cyanidin on rankl-induced differentiation and fusion of osteoclasts. J Cell Physiol 2016;231(3):558-567

19 Perron NR, Brumaghim JL. A review of the antioxidant mechanisms of polyphenol compounds related to iron binding. Cell Biochem Biophys 2009;53(2):75-100

20 Sheweita SA, Khoshhal KI. Calcium metabolism and oxidative stress in bone fractures: role of antioxidants. Curr Drug Metab 2007;8(5):519-525

21 Kenkre J, Bassett JH. The bone remodelling cycle. Ann Clin Biochem 2018;55(3):308-327

22 Alhasyimi AA, Rosyida NF. Cocoa administration may accelerate orthodontic tooth movement by inducing osteoclastogenesis in rats. Iran J Basic Med Sci 2019;22(2):206-210 